\title{
Simultaneous Use of Elastic and Electromagnetic Waves for the Detection of Buried Land Mines
}

\author{
Waymond R. Scott, Jr. ${ }^{a}$, Gregg D. Larson ${ }^{\mathrm{b}}$, and James S. Martin ${ }^{\mathrm{b}}$ \\ ${ }^{a}$ School of Electrical and Computer Engineering \\ ${ }^{\mathrm{b}}$ School of Mechanical Engineering \\ Georgia Institute of Technology \\ Atlanta, GA 30332
}

\begin{abstract}
A hybrid technique has been developed that uses both electromagnetic and elastic waves in a synergistic manner to detect buried land mines. The system consists of a moving electromagnetic radar and a stationary elastic-wave source. The source generates elastic waves in the earth. These waves interact with the buried mine and cause both the mine and the earth to be displaced. Because the mechanical properties of the mine are different from those of the earth, the displacements in the region of interaction are distinct from those associated with the free-field propagation of the waves. The radar is used to detect these displacements and, thus, the mine. Initial investigations have demonstrated the feasibility of this scheme under controlled conditions. The current experimental effort is focused on understanding and overcoming the issues associated with using the system in field conditions.
\end{abstract}

Keywords: land mine, mine detection, acoustic, ground penetrating radar, GPR

\section{INTRODUCTION}

Seismic/elastic techniques show considerable promise for the reliable detection of all types of buried mines, even low-metal anti-personnel mines. The reason for this is that mines have mechanical properties that are significantly different from soils and typical forms of clutter. For example, the shear wave velocity is approximately 20 times higher in the explosive and the plastics used in typical mines than in the surrounding soil. In addition, mines are complex mechanical structures with a flexible case, a trigger assembly, air pockets, etc. The complex structure gives rise to structural resonances, non-linear interactions, and other phenomenology that is atypical for both naturally occurring and man-made forms of clutter. Thus, this phenomenology can be used to distinguish a mine from clutter.

A system has been developed at Georgia Tech that uses a radar based displacement sensor for the local measurement of seismic displacements without physically contacting the soil surface [1-3]. The non-contact nature of this sensor makes the system capable of interrogating the soil surface near or immediately above a mine. This substantially increases the measurable effects of the mine's presence over schemes which rely on elastic waves scattered by the mine to propagate to a remote sensor location. Figure 1 depicts the present system configuration. The system consists of the electromagnetic radar and the seismic source. The source (an electrodynamic shaker coupled to the ground by a narrow foot) preferentially generates an elastic surface (Rayleigh) wave in the earth. The Rayleigh wave causes both the mine and the surface of the earth to be displaced as it propagates past the mine. Since the amplitude of Rayleigh wave displacements decreases exponentially with depth, only the soil near the surface is interrogated for the presence of mines. The depth of soil which is examined is a function of the frequency of the source. For typical mine depths and sizes, this is in the 100 to $1,000 \mathrm{~Hz}$ range. The motion of the mine is different from the surrounding soil, because the elastic properties of the mine are quite different than those of soil. The displacement of the surface of the earth when a mine is present is different than when it is not present because of the local and propagating waves scattered by the mine. The 
electromagnetic radar is used to detect these displacements and, thus, the mine. This idea of using elastic and electromagnetic waves synergistically has been proposed previously [4-6], but it has not been seriously investigated until now.

The radar based displacement sensor has been designed and built to measure small vibrations of the soil and the mine. The sensor radiates electromagnetic waves toward the earth. These waves are reflected from the surface of the earth and the mine, where they are amplitude and phase modulated by the transient displacements of the earth and the mine. The reflected waves are received and demodulated. The resulting demodulated signals are proportional to the surface displacement. The radar can measure vibrations as small as $1 \mathrm{~nm}\left(10^{-9} \mathrm{~m}\right)$ as currently configured. The end of the wave guide (which functions for both transmit and receive) illuminates an area on the earth's surface comparable to its own cross section $(1 \mathrm{~cm} \times 2 \mathrm{~cm})$ over which the displacement is integrated.

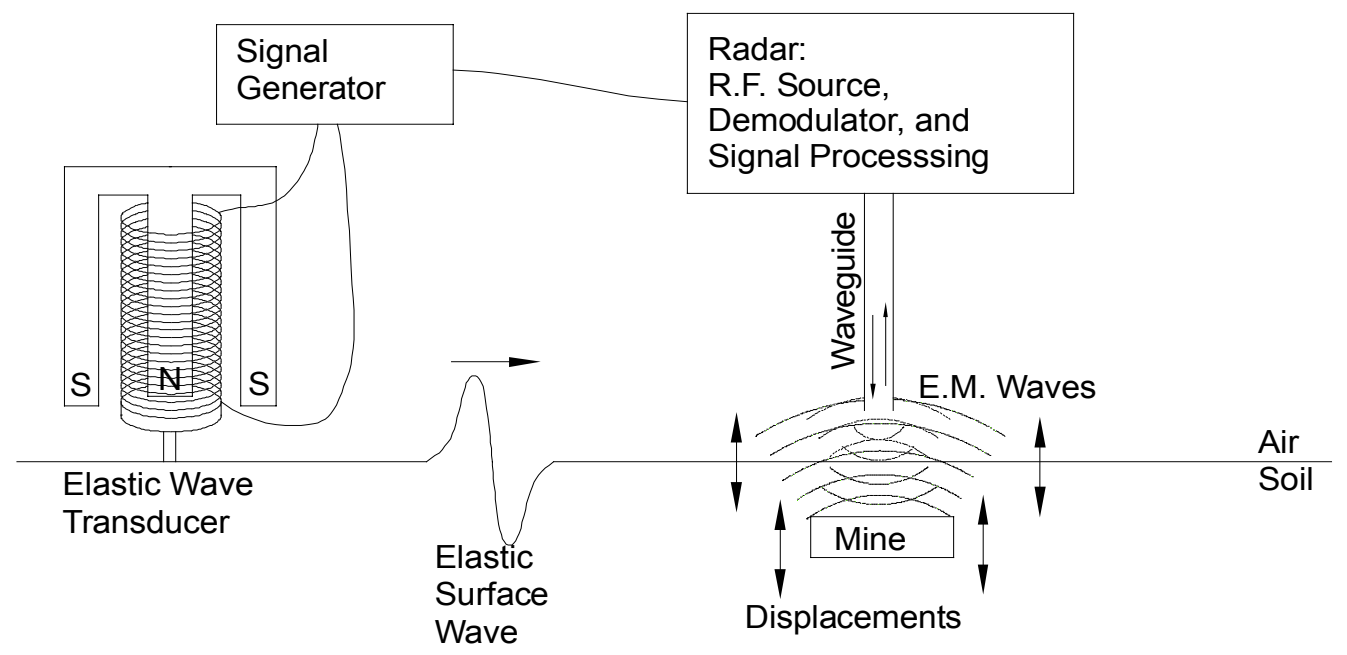

Figure 1: Diagram of the hybrid elastic and electromagnetic mine detection system.

The system is currently being studied in a laboratory scale experimental model. The model, which is depicted in figure 2, consists of a wedge shaped tank filled with over 50 tons of damp compacted sand to simulate soil. The seismic source is located near the tip of the wedge and is bi-directive toward the search area and the back wall. Simulated mines, inert mines, and clutter, such as rocks and sticks, are buried within a $2 \mathrm{~m} \times 2 \mathrm{~m}$ region in the center of the tank. The radar can be scanned above this region with a three degree of freedom positioner fixed above the tank.

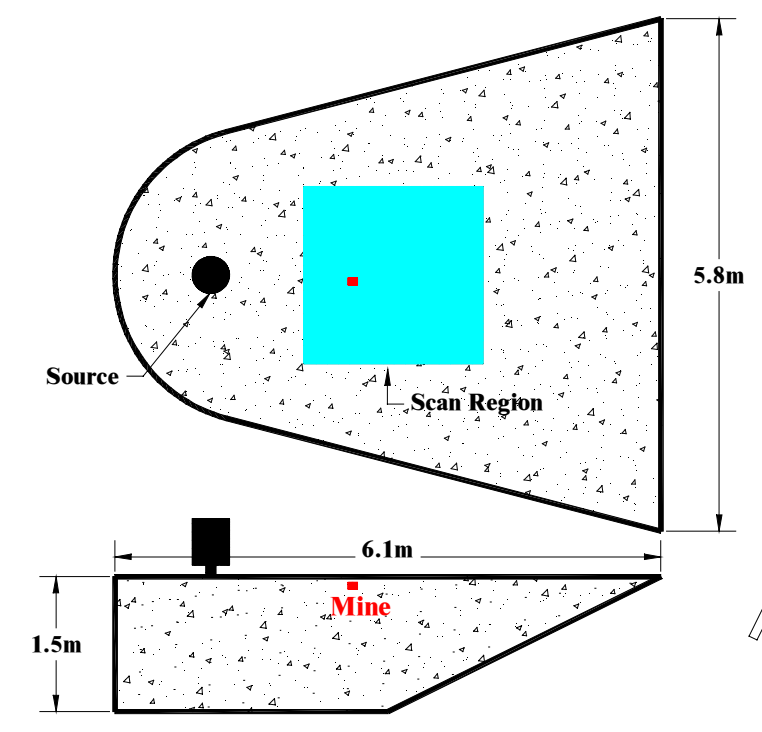

Figure 2: Experimental model for mine detection 
Previously presented work with this experimental model and a previous experimental model has demonstrated the feasibility of the elastic / electromagnetic mine detection concept [7]. Current efforts with the model have focused on resolving critical issues for the refinement of the concept into a field operable mine detection system. Recent experimental work has sought to answer the following questions: Does the presence of clutter and other targets unacceptably degrade system performance? Does surface vegetation degrade the radar sensor's performance to make mine imaging impossible? Is the current 100 to $1000 \mathrm{~Hz}$ frequency range used in the system sufficient for the expected range of mine burial depths? Can the same system configuration and parameters be used for the detection of both anti-personnel (AP) and anti-tank (AT) mines? Can a source be constructed which can be translated along with the sensor for continuous rather than incremental scanning?

\section{EFFECTS OF BURIED CLUTTER AND MULTIPLE TARGETS}

The primary detection cues used for all of the AP mine types which have been studied in the experimental model have been resonances of the mine case, trigger mechanism, and overlying soil [6-8]. These are excited by the passage of the Rayleigh wave and characterized by large displacements which persist after the passage of the incident pulse. Although mines exhibiting resonances scatter a larger propagating wave field than similarly sized non-resonant objects, the most pronounced feature of the field scattered by these mines is its mostly local nature. This appears to result from a mostly reactive soil loading. For the mine types studied thus far, the localized resonant motion has been an excellent indicator of a mine's location and extent.

Imaging of mines from multiple surface displacement measurements can be done in many ways. The current imaging scheme involves a multi-step process which filters forward travelling waves (those components directed away from the source) out of the data in the wavenumber domain leaving the reflected waves and a portion of the non-propagating waves. The energy in these remaining waves at times near the time of arrival of the incident wave is assigned to each measurement point forming an image. While there is certainly room for refinement of this algorithm, it does provide an objective method for the generation of mine field images which seem to incorporate the subjective observations of resonant mine behavior. Unlike background subtraction, this algorithm does not rely on information which would be unavailable to a mine detection system operating in the field. It is therefore, a reasonable (but not a certain) candidate for use in a field ready system. Previous work has shown that this technique can be used to image several types of AP mines and distinguish them from non-resonant buried clutter [7,9].

An experiment was performed to address the issue of imaging a minefield containing multiple candidate mine targets, some actual and some false. For this study, the false targets were four mine-sized rocks. The actual targets were four inert AP mines of different types and one radar mine simulant. The 5 mines and 4 rocks were buried at depths of one to three centimeters within a $120 \mathrm{~cm}$ by $80 \mathrm{~cm}$ search area. The layout of this experiment and the relative scale of the buried objects is shown in figure 3 . The goal was to determine whether the presence of the false targets or the scattered fields of the actual targets would obscure the apparent size or location of any of the actual targets in the image which could be formed of the minefield. Also of concern was the potential that multiple scattering would produce ghost images of some of the mines or that the signatures of the most identifiable AP mines (the TS-50 and VS-50) might dictate the dynamic range of the resulting image so as to mask the presence of the least identifiable mines (the M-14 and SIM-9). The image formed of this search area is shown in figure 4. It is clear from the figure that all 5 mine locations have been correctly depicted and that no false images were generated. The image of the VS-50 mine has been elongated in the direction of the butterfly mine which was buried behind it. This is an artifact of the technique used to filter out the forward traveling waves and can be fixed by improving the imaging algorithm. It is obvious from the nature of the elastic / electrodynamic system that false targets could have been selected that would have been problematic for this type of imaging. Rocks were selected because they are naturally occurring and ubiquitous. Resonant false targets are neither and should, therefore, not constitute an unacceptable false alarm rate. 


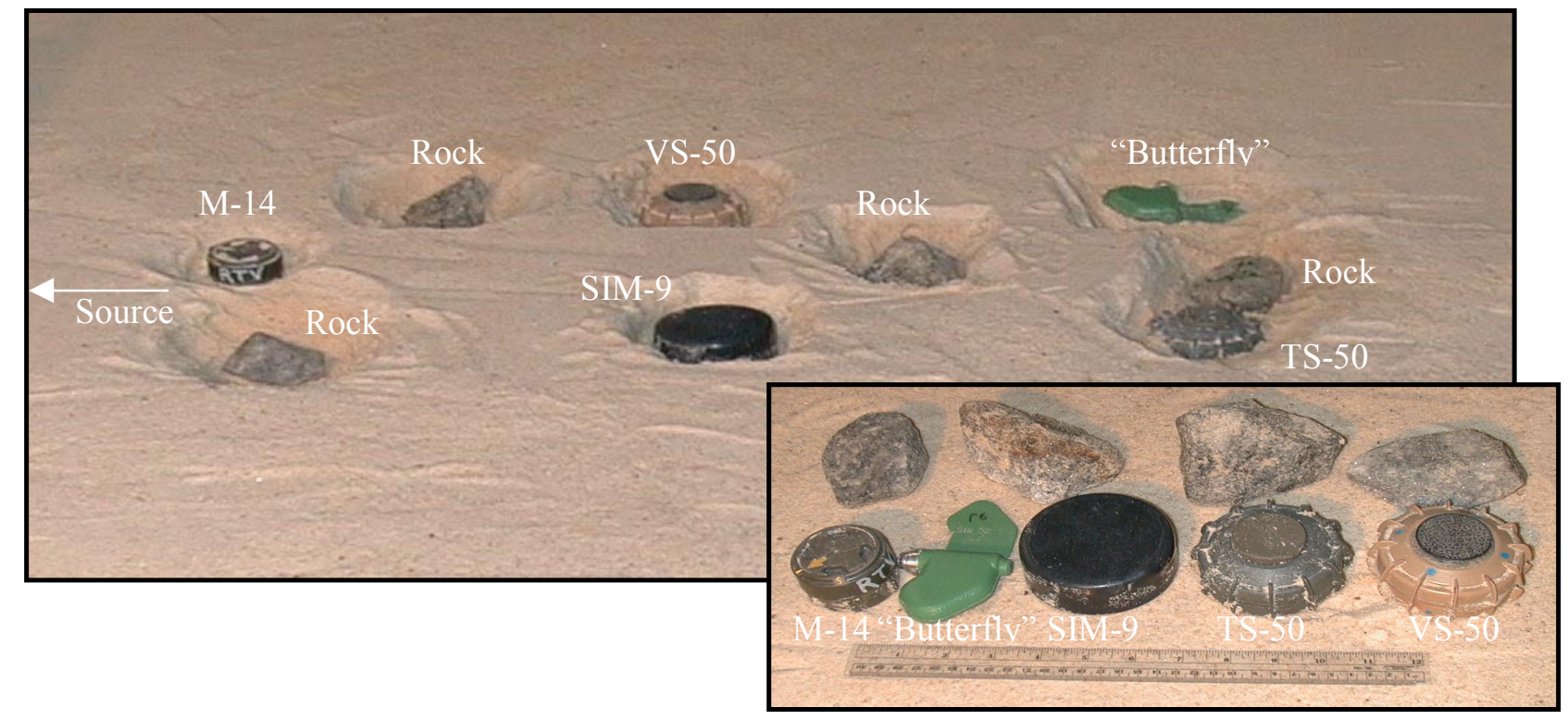

Figure 3: Mines and rocks in buried clutter experiment

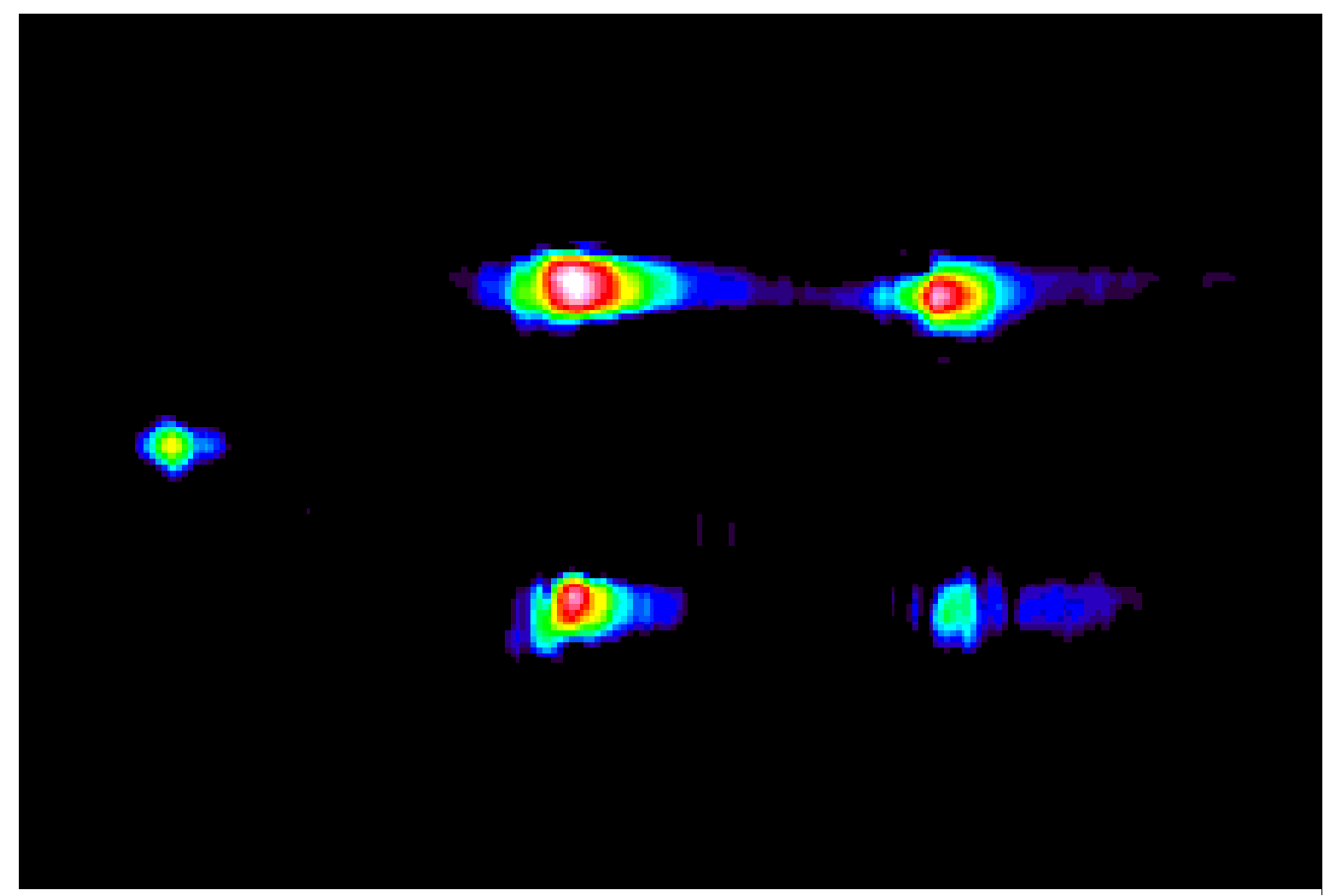

Figure 4: Image formed of minefield in buried clutter experiment 


\section{TRANSLUCENT SURFACE COVER}

In early testing of the elastic / electromagnetic mine detection system, the surface of the soil surrogate used in the experiments was maintained level, smooth, and bare. This provided the radar sensor with a seismically modulated EM reflection from the surface that was nearly optimal (large and uncorrupted). In the field, the surface will be rough and lie under some type of ground cover. The ability of the system sensor to see through common surface cover such as grass or light vegetation is essential for practical system operation. Some types of ground cover, obviously, will be opaque to the radar's interrogation signal. These will require special consideration and possibly an alternate sensor design. Standing water is an example of such a problematic case. In general, vegetation is likely to be translucent to the radar sensor with a portion of the EM reflection originating in the vegetation and the remainder coming from the surface below. The signal which is reflected from the vegetation can corrupt the total return in two ways. First, it will reduce the electromagnetic signal component reflected from the surface. This will reduce the level of the measured displacement signal. Second, any motion of the vegetation will produce an additional modulation of the carrier unrelated to the motion of the underlying surface. This will increase the effective noise floor. To test the ability to penetrate surface cover, pine straw was selected both for the convenience of its application and because it is a commonly occurring ground covering. In the experiment, a $2.3 \mathrm{~cm}$ layer of pine straw was spread over the surface beneath which a TS-50 AP mine was buried along with 4 mine sized rocks and two sticks. The layout of this experiment can be seen in figure 5 .

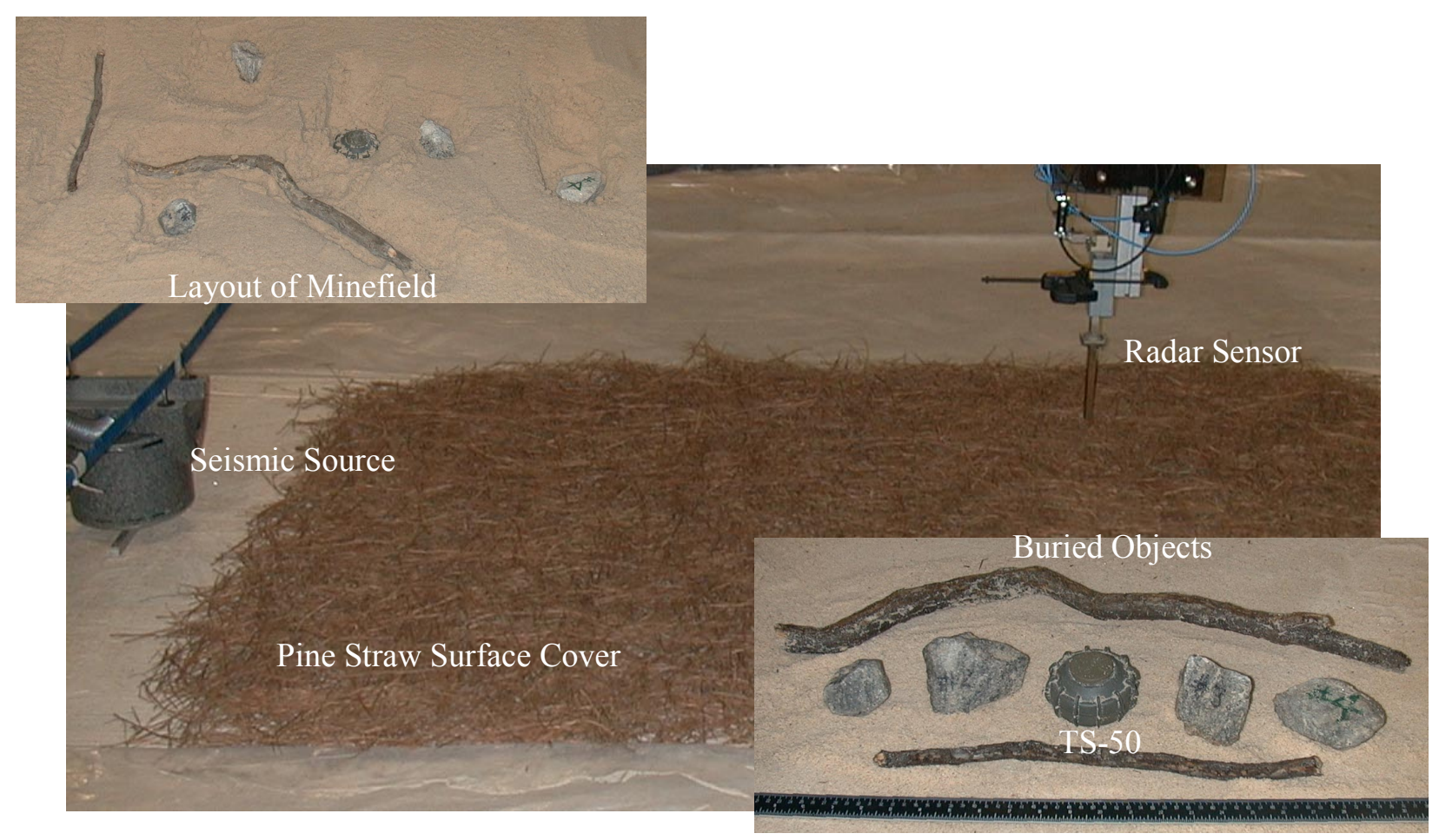

Figure 5: Surface Vegetation Experimental Setup

Figure 6 shows the image formed from the pine straw covered surface. The location and extent of the TS50 mine are apparent. There is less contrast in this image than for similar images formed in the absence of surface covering. An examination of the time domain signals which contribute to the image reveals that the dominant effect of the pine straw was to force an increase in the seperation between the antenna and the ground's surface which resulted in a reduced signal level and a loss of some spatial resolution. When this is corrected for, the only direct effect of the surface cover that can be observed is a slight increase in the spatial noise floor (signal variability with range). This is attributable to variations in the received EM surface reflection which is reduced by up to $6 \mathrm{~dB}$ when the pine straw is present. 


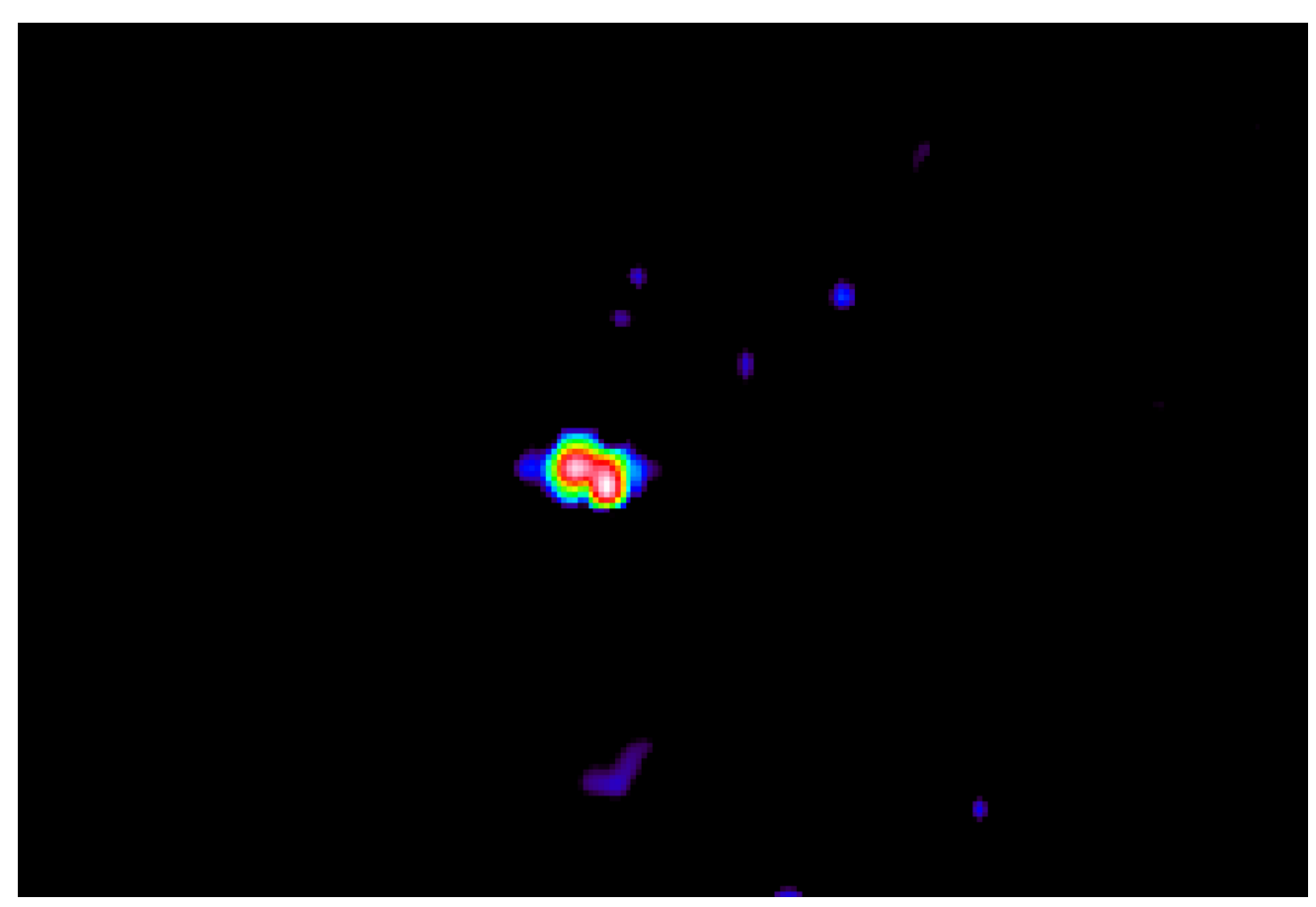

Figure 6: Image Formed of Pine Straw Covered Mine Field

\section{MINE SIZE AND DEPTH RANGE OF DETECTABILITY}

There are two ranges of mine size and burial depth which are of concern for the mine detection problem. The first of these is for AP mines which are typically a few hundred milliliters in volume and buried such that the mine trigger is a few centimeters below the soil surface. AP mines were the focus of most of the early work on the elastic/ electromagnetic mine detection system because they are usually perceived as a more difficult problem. The efficacy of a system like this for AP mine detection does not, however, automatically imply success in the detection of AT mines. These mines are typically several liters in volume and have burial depths up to a few decimeters. Since Rayleigh waves penetrate the surface to a depth proportional to their wavelength it is likely that lower frequencies would be required for the detection of AT mines. Since the detection of AP mines was greatly facilitated by resonances of the mines, it is unlikely that the AT mine problem is simply a direct scaling of the AP mine imaging technique. This would imply a system bandwidth down to 10 or $20 \mathrm{~Hz}$ which is not possible with the current experimental model, but should be possible in a system that operates in the field. Currently, the operating band of the system $(100-1,000 \mathrm{~Hz})$ is dictated by source response, ambient noise, and reverberation in the experimental model at low frequencies. Attenuation in the soil surrogate limits the bandwidth at high frequencies.

Two types of inert AT mines were tested: VS-1.6 and VS-2.2. These mines are shown in figure 7 along with a TS-50 AP mine for size comparison. Both AT mines have plastic cases and spring loaded piston triggers. The triggers are considerably stiffer than those on the AP mines which were previously tested. The results for both mines were quite similar. Figures 8 and 9 show seismograms generated by one-dimensional scans over the two AT mines as a function of mine burial depth. The data was processed in a slightly different way from the AP mine scans in that the pulse shape which was used had a center frequency that was an octave lower ( $225 \mathrm{~Hz}$ as opposed to $450 \mathrm{~Hz}$ for the AP mine scans). The experimental data used to reconstruct either pulse response are identical. The difference is in the post processing. Both processing techniques could be used simultaneously. The pulse with the lower center frequency was chosen for the AT mine in order to emphasize the effects of the spectral components which penetrated the soil to a sufficient depth to interact with the mine. 
From data in figures 8 and 9 the presence of the mine is apparent up to a depth of about $10 \mathrm{~cm}$. Above the mine, an amplification of the incident can be observed at 2, 4, and $6 \mathrm{~cm}$ depths. Propagating waves that have been reflected from the mines are clearly apparent at 4 and $6 \mathrm{~cm}$ depths. The strength of the reflected field at these depths appears to be inversely related to the duration of the localized motion above the mine which indicates that the radiation of Rayleigh waves may constitute a significant source of damping for the mine motion. There is also evidence of dispersion in the soil layer above the mine: the leading edge of the incident signal is clearly delayed as it passes above the mine and the pulse shape and arrival time well beyond the mine are quite different than they are at the same location for the no mine case.

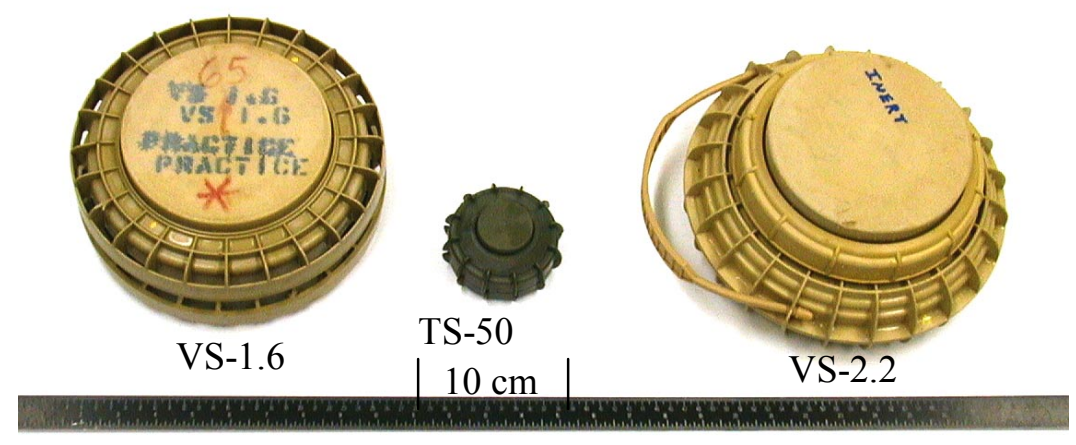

Figure 7: Size Comparison of AT Mines and AP Mine

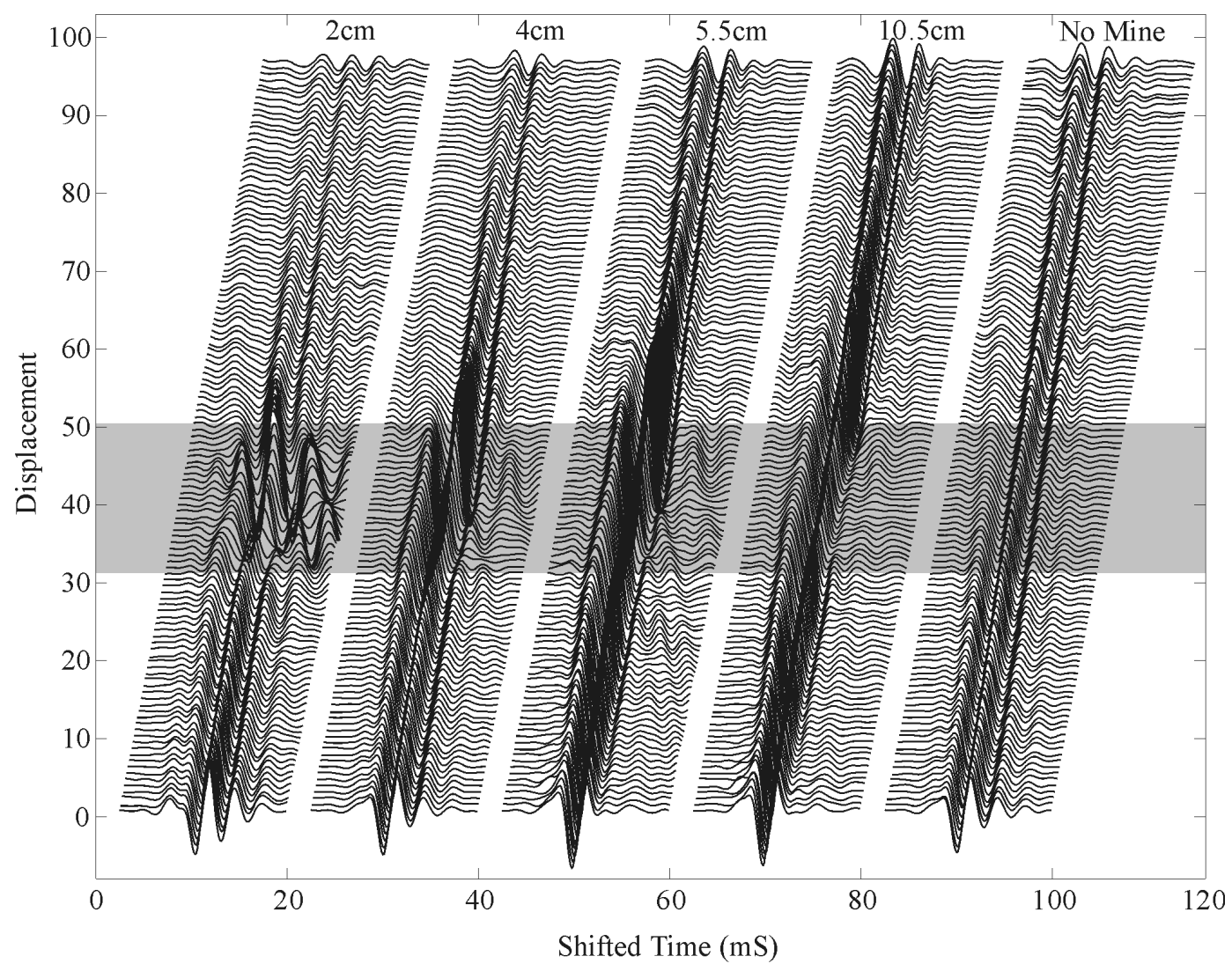

Figure 8: 1-D Scans of VS 2.2 AT Mine at Various Depths 


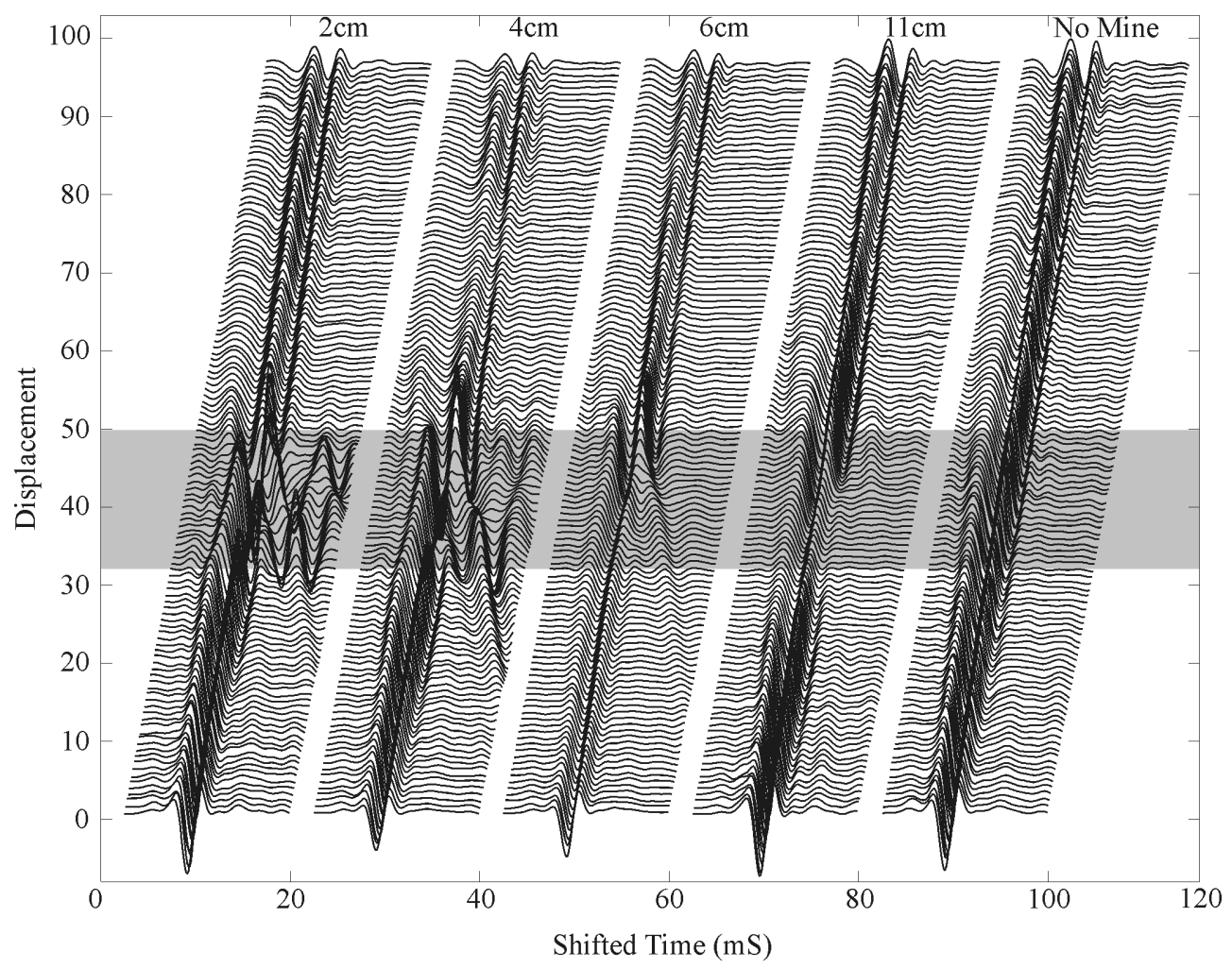

Figure 9: 1-D Scans of VS 1.6 AT Mine at Various Depths

In the seismograms of figure 8 there is very little sign of the AT mine at the $11 \mathrm{~cm}$ burial depth. Its presence is, however, much more apparent if the forward travelling waves are removed from the seismograms. This is because the forward travelling waves are the dominant component of the receive signal but are only slightly effected by the presence of the mine. In contrast, the smaller reverse propagating and standing waves are related almost entirely to the mine and other scatters in the search area. The seismograms of figures 10 and 11 demonstrate this. These have been generated by filtering the forward propagating components out of the data depicted in figures 8 and 9. A gain factor, which is proportional to mine depth, has been applied to force the traces onto comparable vertical scales. In the imaging process, where target depth is unknown, a logarithmic scale is used for this purpose. Where no mine was present the gain factor for the deepest corresponding burial was used to demonstrate the effective noise floor of this technique. These figures demonstrate that, using the current system and model, an AT mine could be imaged to a depth of 10 or 11 centimeters without substantial computational effort. This is, by no means a limit on the detection technique. The spectral content of the incident and the processing technique could be improved to extend this further. Acausal artifacts of the filtering operation which elongate the mine signature can be seen in the filtered seismograms. This is clearly one area in which the algorithm can be improved. The artifacts appear as wavefronts that originate at the mine but arrive instantaneously in the region behind the mine. Similar artifacts in the direction of the source are obscured by the Rayleigh wave reflected by the mine. Conceptually these should be precluded by the processing algorithm but the arise because of the spatial windowing of the scan region and the abrupt edge of the filtering function in the wavenumber domain.

In order to test the feasibility of imaging a deeply buried AT mine a 2-dimensional scan was performed with the VS-1.6 buried $11 \mathrm{~cm}$ deep. The imaging technique was identical to that used for the AP mines, but incorporated the lower center frequency pulse. The image which was formed is depicted in figure 12. The location and extent of the mine are apparent in this image. There is considerably more smearing of this image than for those formed of the AP mines. This is due, in part, to processing artifacts and, in part, to relatively large propagating wave reflections associated with the AT mine. 


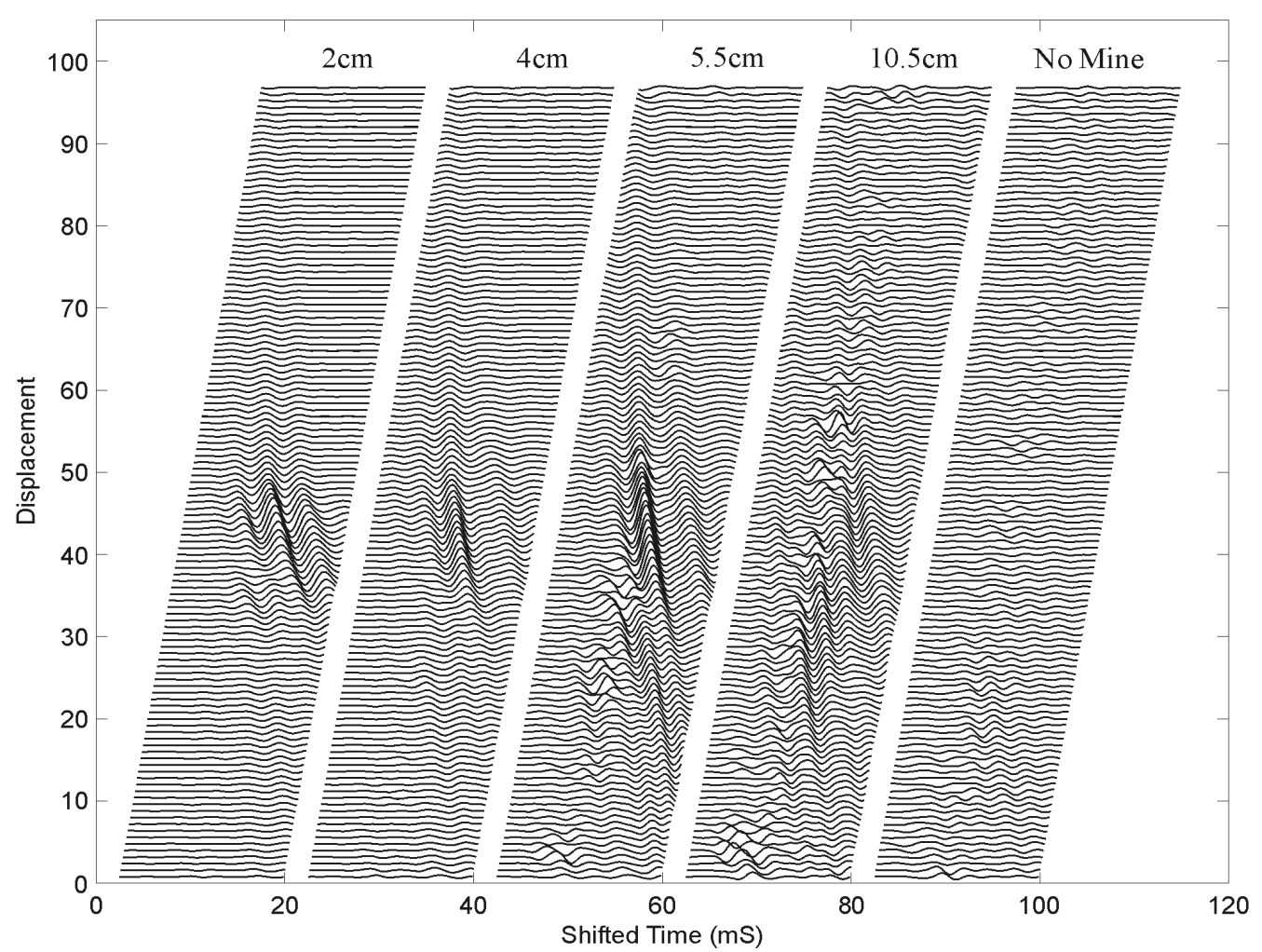

Figure 10: 1-D Scans over VS-2.2 AT Mine with Forward Waves Filtered Out

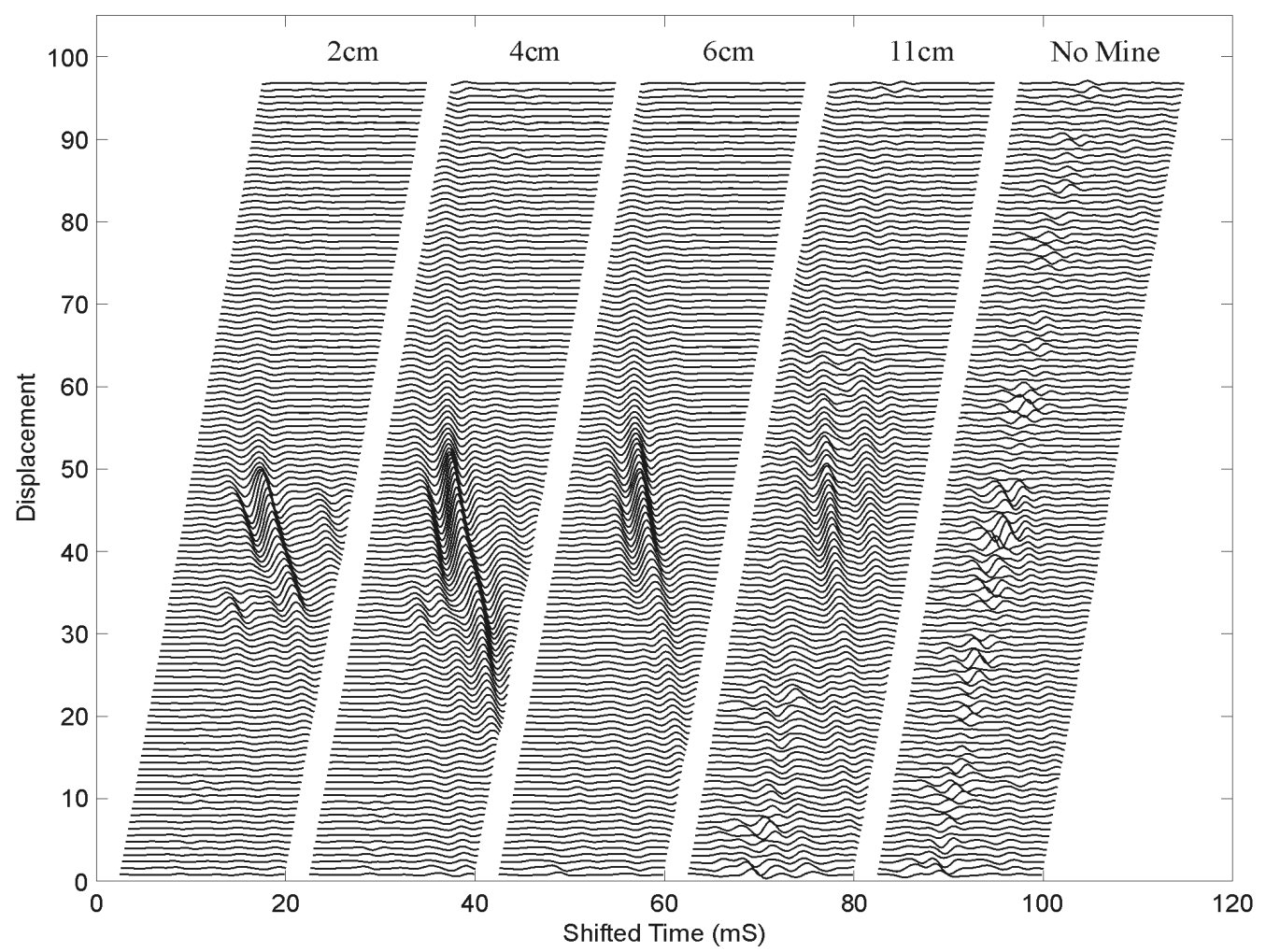

Figure 11: 1-D Scans over VS-1.6 AT Mine with Forward Waves Filtered Out 


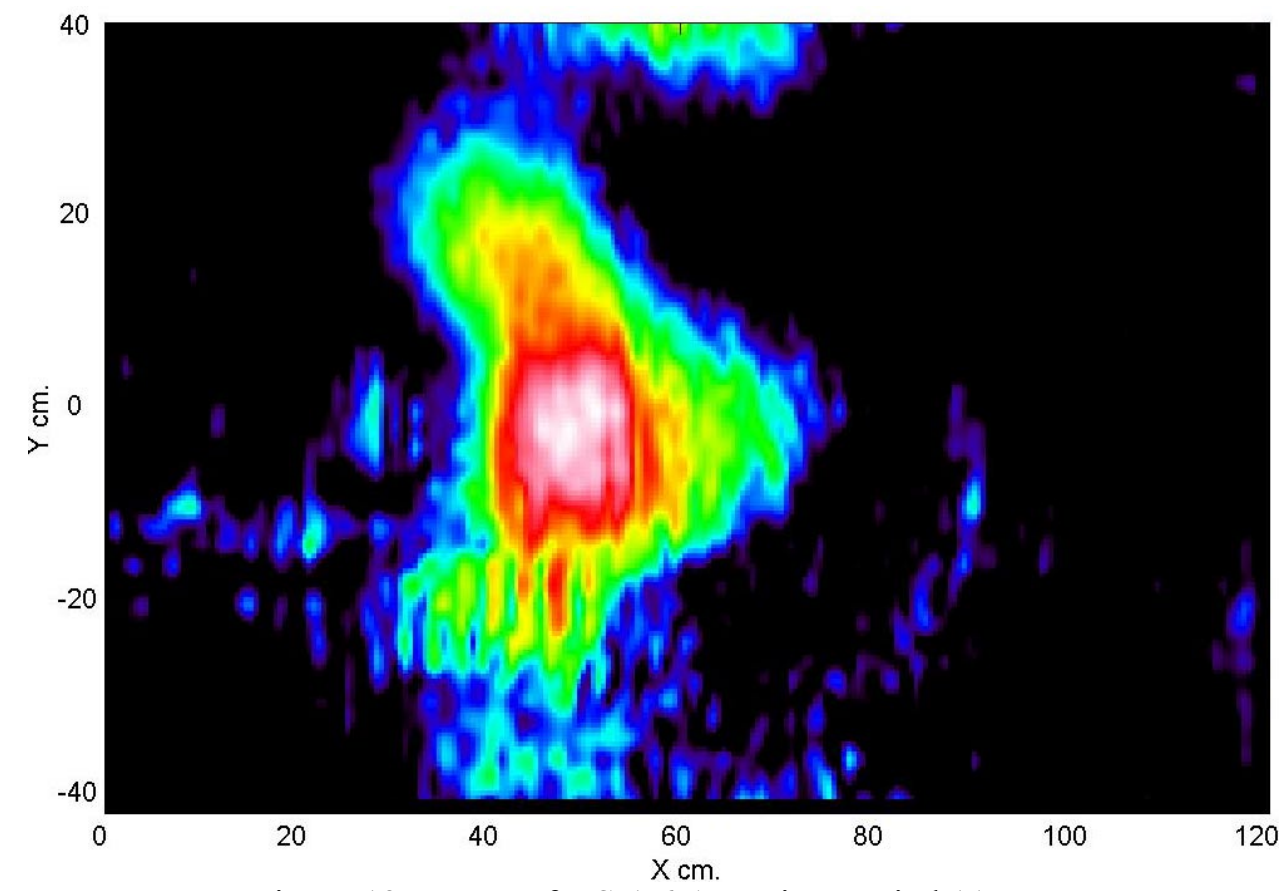

Figure 12: Image of VS-1.6 AT Mine Buried $11 \mathrm{~cm}$ Deep

\section{SCANNABLE SEISMIC WAVE SOURCE}

The electrodynamic shaker, which is currently being used as a seismic source, was selected for its compatibility with the experimental model. It provides a large broadband seismic excitation and does not couple energy into the sensor by any path other than the propagation of elastic waves through the ground. In this regard, it has been extremely well suited to the design of system components and the evaluation of system capabilities. It may, however, not be the best source design for a system which will operate in the field.

For a field operable mine detection system the seismic source needs to be relocated in either discrete increments or continuously during the search process. A reproducible source to surface coupling at each transmitting location is a desirable feature of such a source because it obviates the need to compute an additional transfer function in order to assemble a minefield image. The current seismic source is difficult and time consuming to move. It also does not generate a surface wave that is entirely reproducible when the source relocated. This has been observed even in the benign environment of the laboratory experimental model. The shaker design could be modified for use in a field operable system if the source were coupled to the ground through a rolling point of contact. This design has yet to be explored experimentally, but it is by no means the only option. Several alternative source designs have been proposed. The simplest of these are currently being tested in the experimental model.

Other authors have noticed that it is possible to couple roughly a thousand times more energy into soils from airborne sound than the large density mismatch at the air soil interface would indicate. This is, in part, because the elastic wave speeds in typical soils are considerably lower than the sound speed in air. Although, this observation seems promising for the system under development, an unfortunate consequence of the low propagation speeds is that soils excited by airborne sound respond in a locally reactive way to the excitation [10]. This, by implication, precludes the effective generation of Rayleigh waves (which propagate only along the soil surface) with airborne sound. In order to overcome this apparent limitation a surface excitation is required with substantial non-radiating wavenumber components in the air above the soil. This can be accomplished if the soil surface is in the near field of an aero-acoustic source. 
The experimental aero-acoustic seismic source is a 10" moving coil loudspeaker which is suspended 1 to $3 \mathrm{~cm}$ above the surface of the sand. Initial testing indicates that the speaker must be well within a seismic wavelength of the surface to effectively couple energy into the Rayleigh wave. At large distances from the surface there is no angle of incidence at which the airborne wave can match the phase velocity of the much slower Rayleigh wave. Measurable displacements can still be excited at these large separations, but they are due almost entirely to energy coupling into downward directed compressional waves. The trace velocity measured for the pulse along the surface under these circumstances is exactly the propagation speed in air. Figure 10 shows a seismogram of arrivals generated by translating the aero-acoustic source at a height $1 \mathrm{~cm}$ above the soil surface with respect to a fixed accelerometer receiver for a $1 \mathrm{~m}$ transit. The spectrum of the received signals is comparable to that obtained using the surface-coupled shaker source although colored toward the lower frequencies. The displacements are within the measurable range of the existing radar based sensor and it is not unreasonable to believe that they could be made comparable to the current shaker source had a more powerful aero-acoustic source been used. Generally, the aero-acoustic source is more effective at lower frequencies because of the relatively large area beneath the speaker to which a uniform excitation can be applied. An accelerometer was used for these measurements rather than the radar sensor because it provides an absolute measure of displacement. Backside motion introduced by either mechanical or airborne acoustic coupling directly into the radar sensor is problematic for this sort of measurement and will be addressed in future work.

In addition to the two source designs mentioned above many other seismic sources are under consideration for the field operable system. These include thermoelastic laser and microwave sources, air and water jets, electrical arc sources, and ultrasonic parametric sources. Each of these options have intrinsic tradeoffs when compared with other designs. The laser, for example, would allow for very large standoff distances from the earth's surface but would be substantially more expensive and less powerful than the sources considered here.

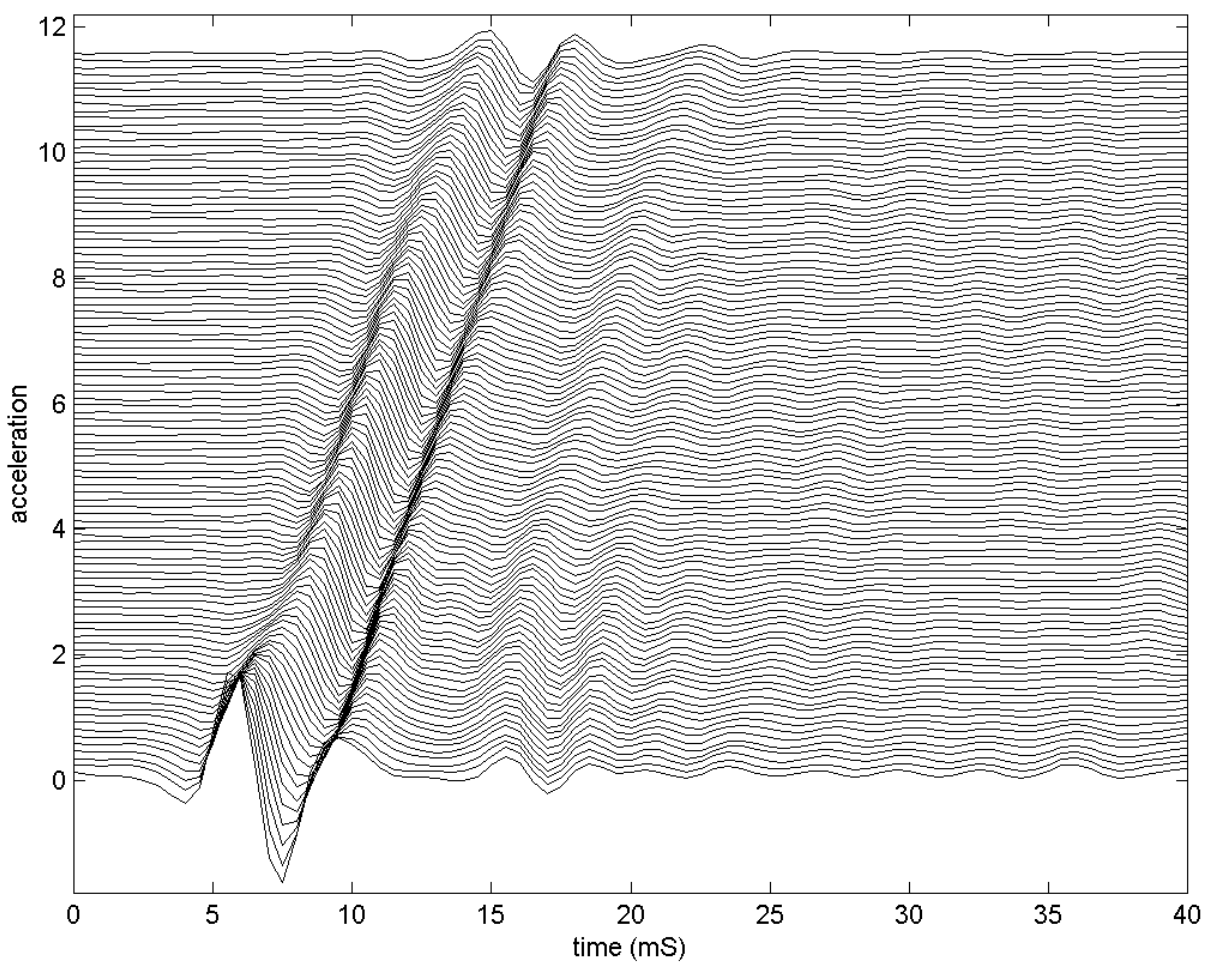

Figure 13: Rayleigh Wave Generated with an Aero-Acoustic Source 


\section{CONCLUSIONS}

The elastic / electromagnetic land mine detection technique has been tested under laboratory conditions which mimic a variety of realistic mine detection scenarios. None of these conditions appear to be significant hurdles for the development of a field operable mine detection system.

A prototype mine detection system has been demonstrated which is capable of detecting inert AP and AT mines and distinguishing them from buried clutter. The efficacy of the system has been demonstrated over a range of anticipated burial depths with both bare ground and ground covered with thin vegetation. It has been shown that multiple mine targets in close proximity do not pose a significant imaging problem. A Rayleigh wave source has been successfully tested which does not require direct ground contact and can be scanned over the surface of the ground in field operations. Integration of the new source into the detection system, evaluation of alternative source and sensor designs, testing additional mine types, and addressing problematic operating environments will be the focus of future work on the development of the system.

\section{ACKNOWLEDGEMENTS}

This work is supported in part under the OSD MURI program by the US Army Research Office under contract DAAH04-96-1-0448

\section{REFERENCES}

1. Scott, W.R., Jr., Schroeder, C., and Martin, J.S., "An Acousto-Electromagnetic Sensor for Locating Land Mines," SPIE, AeroSense, Detection and Remediation Technologies for Mines and Minelike Targets III, Orlando, FL, pp. 176-186, April 1998

2. Scott, W.R., Jr. and Martin, J.S., "An Experimental Model of an Acousto-Electromagnetic Sensor for Detecting Land Mines," Proceedings of the 1998 IEEE Antennas and Propagation Symposium, Atlanta, GA, pp. 978-83, June 1998.

3. Scott, W.R., Jr., Schroeder, C., and Martin, J.S., "A Hybrid Acoustic/Electromagnetic Technique for Locating Land Mines," Proceedings of the 1998 International Geoscience and Remote Sensing Symposium, Seattle, Washington, July 1998

4. C. Stewart, "Summary of Mine Detection Research," U.S. Army Technical Report 1636-TR, Vol. 1, May 1960.

5. G.S. Smith, "Summary Report: Workshop on New Directions for Electromagnetic Detection of Non-Metallic Mines," Report for U.S. Army BRDEC and ARO, June 1992

6. C. Stewart, Summary of Mine Detection Research, Vol. I, pp. 172-179, Tech. Report 1636-TR, May 1960, U.S. Army Engineering Res. and Devel. Labs, Corps. of Eng., Belvoir, VA.

7. Scott W.R., Jr. and Martin J.S., "Experimental Investigation of the Acousto-Electromagnetic Sensor for Locating Land Mines," Proceedings of the SPIE: 1999 Annual International Symposium on Aerospace/Defense Sensing, Simulation, and Controls, Orlando, FL, Vol. 3710, pg. 204-14, April 1999.

8. Scott, W.R., Jr., Schroeder, C.T., and Martin, J.S., "An Acousto-Electromagnetic Method for Detecting Buried Objects," Proceedings of the XXVI th General Assembly of the International Union of Radio Science, Toronto, Canada, pg. 724, August 1999

9. Behboodian, A., Scott, W.R., Jr. and McClellan, J.H. "Signal Processing of Elastic Surface Waves for Localizing Buried Land Mines," Proceedings of the 33rd Assilomar Conference on Signals, Systems, and Computers, Assilomar, CA, October 1999

10. Sabatier, J.M. et.al., "Acoustically Induced Seismic Waves", Journal of the Acoustical Society of American Vol. 80 \#2, pg. 646-9, August 1986 